\title{
Spektrum der Refraktiven Chirurgie 2019
}

\author{
Gernot Steinwender
}

(C) Springer-Verlag GmbH Austria, ein Teil von Springer Nature 2019

Das Ziel der refraktiven Chirurgie ist die dauerhafte Korrektur von Brechkraftfehlern, welche die weltweit häufigste Ursache für eine reversible Seheinschränkung darstellen. Durch die steigende Prävalenz der Myopie mit geschätzten Milliarden betroffenen Patienten und Patientinnen bis zum Jahr 2050 werden Brechkraftfehler auch zunehmend $\mathrm{zu}$ einem relevanten Thema der öffentlichen Gesundheit. Obwohl die refraktive Chirurgie oftmals als kosmetische Chirurgie eingestuft wird, reichen ihre Folgen weit über Brillenunabhängigkeit hinaus und führen nachgewiesenermaßen auch zur Steigerung der Lebensqualität und Verbesserung der Arbeitsfähigkeit.

Zahlreiche Studien konnten in den letzten Jahrzehnten exzellente visuelle Ergebnisse und gute Sicherheitsprofile für traditionelle Laserverfahren wie LASIK (Laser in-situ Keratomileusis) und Oberflächenverfahren (z.B. Phototherapeutische Keratektomie) zur Korrektur von niedrigen und moderaten Brechkraftfehlern nachweisen. Zusätzlich stehen heute für diesen Indikationsbereich auch neuere Verfahren, wie die rein Femtosekundenlaser-basierte Small incision lenticule extraction (SMILE), zur Verfügung. Für die Korrektur von höheren Fehlsichtigkeiten stellen vor allem die Implantation von phaken Intraokularlinsen und der refraktive Linsenaustausch sinnvolle Optionen dar. Vor allem das letztgenannte Verfahren kann durch den Einsatz von Multifokallinsen oder Linsen mit erweiterter Tiefenschärfe auch zur poten- tiellen Korrektur der Presbyopie, die strenggenommen gar keine Form der Ametropie darstellt, angewandt werden.

Bei allen genannten Verfahren kam es in der letzten Dekade zu substantiellen Weiterentwicklungen. So konnten einerseits durch Einführung neuer Bildgebungssysteme zur besseren Patientenselektion und andererseits durch Optimierung der chirurgischen Schritte die Sicherheit und Effektivität dieser Methoden für die Patienten und Patientinnen gesteigert werden.

Die Vielfalt an potentiellen refraktiven Verfahren erfordert jedoch auch eine fundierte Kenntnis der jeweiligen Nutzen-Risiko-Profile, um zusammen mit dem einzelnen Patienten/der einzelnen Patientin die individuell geeignetste Methode auswählen zu können. Aus diesem Grund ist das Ziel der vorliegenden Ausgabe, dem/der interessierten Augenarzt/ Augenärztin einen evidenz-basierten State-of-the-artÜberblick über das sich schnell entwickelnde Feld der refraktiven Chirurgie zu bieten.

\section{Mit besten Grüßen \\ Gernot Steinwender}

Interessenkonflikt G. Steinwender gibt an, dass kein Interessenkonflikt besteht.

Hinweis des Verlags Der Verlag bleibt in Hinblick auf geografische Zuordnungen und Gebietsbezeichnungen in veröffentlichten Karten und Institutsadressen neutral. 